\title{
Analysis of ELVES at the Pierre Auger Observatory
}

\author{
Mussa, R. ${ }^{1 *}$ for the Pierre Auger Collaboration ${ }^{2 * *}$ \\ ${ }^{1}$ INFN Sezione di Torino, via P.Giuria 1, I-10125, Torino, Italy \\ ${ }^{2}$ Observatorio Pierre Auger, Av. San Martín Norte 304, 5613 Malargüe, Argentina
}

\begin{abstract}
In the last six years, the Fluorescence Detector (FD) of the Auger Observatory has been exploited for the study of transient luminous events occuring high above thunderstorms at large distances (250 to more than $1000 \mathrm{~km}$ ) from the Observatory. The first ELVES candidate was discovered during a night shift in 2005, and further studies based on auxiliary subtriggers allowed to modify the third level trigger of the observatory in order to acquire them with reasonable efficiency. This report aims to briefly review the studies underway on the $>4000$ ELVES triggers harvested in the years 2013-18 by the Observatory.
\end{abstract}

\section{Introduction}

The Pierre Auger Observatory is the world's largest infrastructure for the study of the ultra high energy cosmic rays. Besides its main activity, the Observatory has started a program of cosmogeophysics studies, which exploit some of the unique features of its detectors. ELVES are transient luminous events occuring at the base of the ionosphere, when a strong electromagnetic pulse (EMP) is emitted by a lightning. These phenomena, theoretically predicted few years before [1], were photographed for the first time in 1990 from the Space Shuttle [2]. For an observer at ground, ELVES appear as rapidly expanding rings, smoothly fading towards the horizon, and can be observed at distances long enough from the causative lightning bolt to avoid the direct flash to saturate the light sensors.

\section{Triggering ELVES with the Auger FD}

The observation of the ELVES requires a very fast camera with high photon sensitivity either in the close UV band or above $600 \mathrm{~nm}$. The 24 FD telescopes of the Auger Observatory [3], each with a $11 \mathrm{~m}^{2}$ mirror, are ideally suited for this type of observations, but these studies have required a series of changes to the online selection of the events, based on a three-stage trigger.

The first level trigger operates at pixel level with an adjustable threshold that keeps the PMT trigger rate at $100 \mathrm{~Hz}$; at second level (SLT), the trigger searches for track segments made of at least five adjacent pixels which passed the first level trigger. The third level trigger (TLT) is designed to efficiently veto events due to close lightning, featuring a high multiplicity of triggered pixels randomly distributed in time across the camera within the $100 \mu \mathrm{s}$ time window. The multiplicity-based TLT, installed in the

\footnotetext{
*e-mail: mussa@to.infn.it

**http://www.auger.org/archive/authors_2018_09.html
}

Table 1. Number of ELVES triggers taken in 2013-18.

\begin{tabular}{|r|rrr|r|r|}
\hline Year & Single & Stereo & Triplets & Total & notes \\
\hline 2013 & 214 & 83 & 8 & 305 & $4-12 / 2013$ \\
2014 & 425 & 128 & 19 & 572 & \\
2015 & 686 & 117 & 11 & 814 & \\
2016 & 673 & 151 & 21 & 845 & \\
2017 & 906 & 297 & 52 & 1255 & \\
2018 & 527 & 99 & 15 & 641 & $1-9 / 2018$ \\
\hline total & 3431 & 875 & 126 & 4432 & \\
\hline
\end{tabular}

end of 2007 as a replacement of a previous and less efficient version, that allowed the serendipitous discovery of ELVES in our early data sets [4], was modified after a four years long study of prescaled SLT events, which yielded 58 new ELVES candidates [5]. The new TLT performed a check on the angular evolution of the light front around the first triggered pixel. For the sample of pixels of the same column of the first pixel, the algorithm requests that at least two pixels before AND two pixels after the central one have a pulse. Moreover, $80 \%$ of them must show an increasing pulse time. For the sample of pixels of the same row, instead, the algorithm just request three pixels to the left of the central one OR three pixels to the right of it. Any of the two arms must show an increasing pulse time in $80 \%$ of the pixels. In comparison to cosmic rays, ELVES release a large amount of light: therefore, we required at least one pixel with a pulse amplitude greater than 50 ADC counts.

The ELVES trigger was implemented in March 2013 in the FD site of Los Leones, and extended to all FD sites during the following FD shift. During the first year of commissioning, we realised that the standard FD traces, $72 \mu$ s long, did not allow us to see the light emitted from the region of the ionosphere located vertically above the lightning source. Therefore, we modified the FD readout scheme, allowing it to acquire three consecutive frames for 


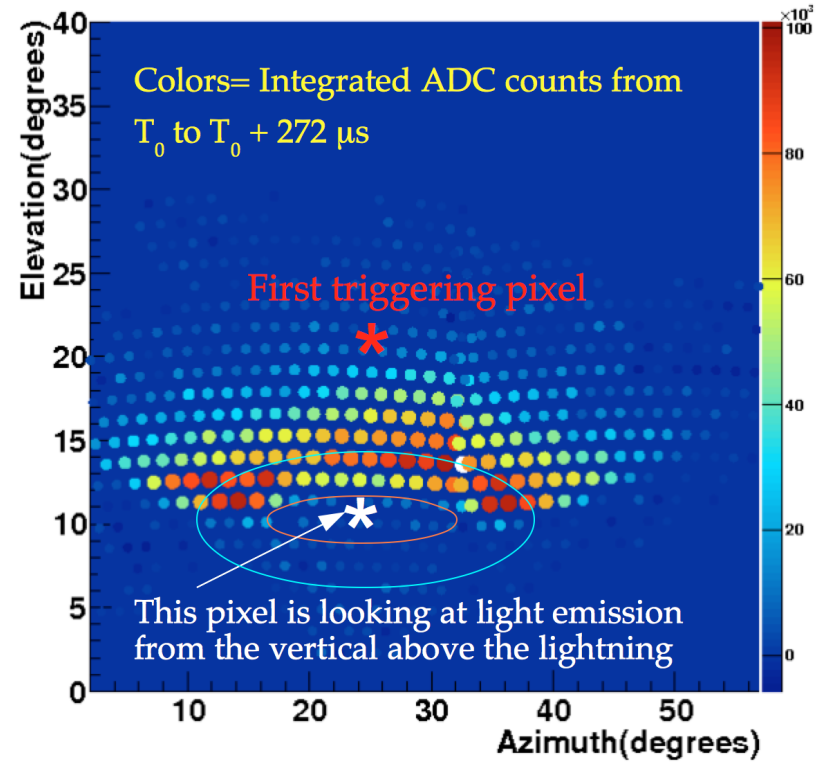

Figure 1. Light distribution integrated in the first $272 \mu \mathrm{s}$.

these special triggers (Extended Readout). This change enables the study of the angular distribution of light emission above the lightning. In particular, the size of the central gap is related to the maximum electron speed in the lightning stroke [6]. The 2014-2016 ELVES dataset has been taken with a maximum trace length of $300 \mu$ s (see Table 1). A typical example of event taken with the extended readout is shown in Fig.1, that displays the distribution of light integrated in the first $272 \mu$ s after the trigger. The vertical above the lightning is represented by a white star, while the red star indicates the first triggered pixel, and the red ellipse indicates the region where the light emission dies down. This choice, though, does not enable us to measure the intensity of light through the full region of maximum emission, outlined by the cyan ellipse in Fig.1.

Fig. 2 shows the time difference between the time of arrival of the light pulse on the central column of the ELVES, as a function of the pixel elevation, and the time of an hypothetical signal traveling at the speed of light along the earth surface. In order to explore the light emission from angular regions at lower elevation angle, it is necessary to largely increase the integration time. Since January 2017, the ELVES trace length has been extended to $900 \mu \mathrm{s}$ (Super Extended Readout scheme) in order to see the full high-intensity region of most events.

\section{Reconstruction of ELVES location and light emission}

The flowchart in Fig.3 shows the steps of the ELVES reconstruction process. First of all, we fit the ADC trace of each pixel to one or more asymmetric gaussians. If

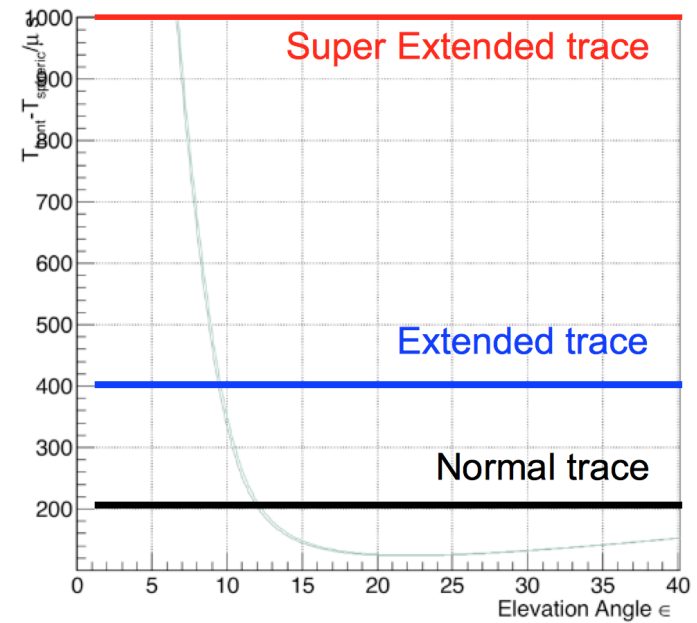

Figure 2. Pulse time vs elevation for the central column of an ideal ELVES: the horizontal bands show the trace length in 2013(black), 2014-16(blue), 2017-2018(red).

less than ten pixels feature a double peak, the reconstruction aims to a precise time and space location of lightning which caused the ELVES, and to a measurement of the angular distribution of the light emission from the base of the ionosphere.

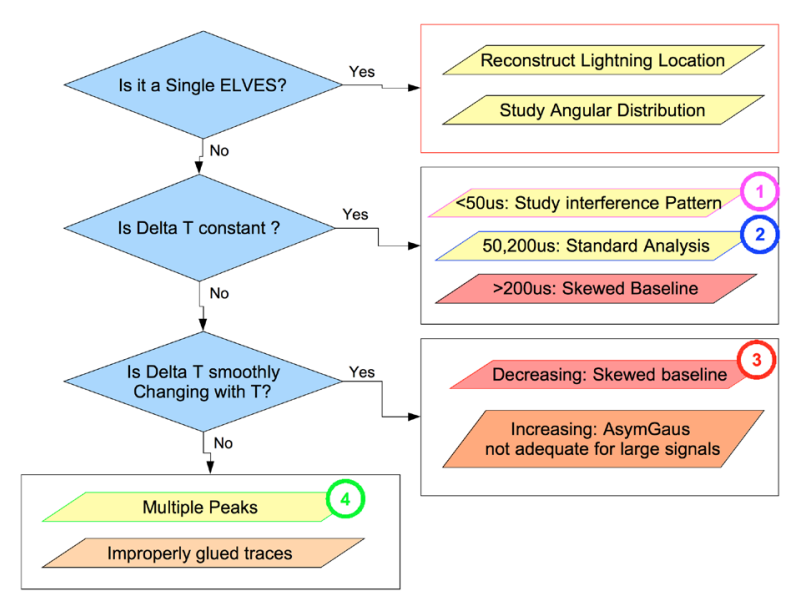

Figure 3. Flowchart of the analysis chain.

The reconstruction of the lightning location is performed in a four-step process:

1. we perform a set of polynomial fits on the pulse times $T_{i}$ of each row and column, to obtain a first rough estimate of the lightning longitude and latitude; the results are inputs to the second fit; 
2. we perform a second fit to minimize the $\chi^{2}=$ $\Sigma_{i=1}^{N}\left(T_{i}-\Delta T_{0}-\overline{O P S}_{i} / c\right)^{2}$ where $\Delta T_{0}$ is the time lag between the emission of the EMP from the source and the observation of the first light at the FD diaphragm, and $\overline{O P S}_{i}$ is the sum of the distances $\overline{S P}$ (from the source to the emission layer) and $\overline{P O}$ (from the emission layer, assumed to have zero thickness and altitude $H_{E M}=92 \mathrm{~km}$, to the FD). The lightning is assumed to be at sea level.

3. the third fit is done releasing the emission altitude $H_{E M}$.

4. the fourth fit is performed allowing also the lightning altitude $H_{B}$ to vary above sea level.

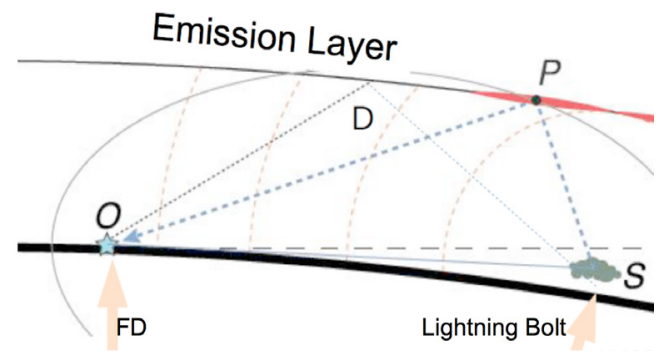

Figure 4. ELVES reconstruction geometry.

After having located the lightning source, in order to calculate the total amount of photons irradiated by the ELVES, it is necessary to transform the light amount observed in each pixel, $P_{i}(F D)$ to the photon surface density $\Phi_{i}=P_{i}(F D) * C_{i}($ geom $) * C_{i}($ atmo $)$ at the base of ionosphere. The geometric correction

$$
C_{i}(\text { geom })=\overline{O P}^{2} /\left(A_{\text {mirror }} A_{\text {emis }}\right)
$$

accounts for the solid angle subtended by the FD diaphragm, and the size of the emitting surface at the base of the ionosphere $A_{\text {emis }}$.

The atmospheric correction:

$$
C_{i}(\text { atmo })=\exp \left(-\left(\tau_{O P, \text { mol }}+\tau_{O P, a e r}\right) * A M(\theta)\right)
$$

accounts for the optical depths $\tau$ of aerosols and molecules through the light path. The airmass $A M(\theta)$ is calculated using the formula in ref.[7].

The corrections (shown in Figs. 5 and 6 for the center of each pixel) are strongly depending on the row number and weakly depending on the column number. After applying these corrections, in the rare case of a perfect dipole, the angular distribution of the emission is azimuthally symmetric with respect to the vertical of the lightning, and the total amount of light emitted from the base of the ionosphere can be easily extrapolated from the observed fraction. More work is needed to model tilted dipoles or more complex emission patterns.

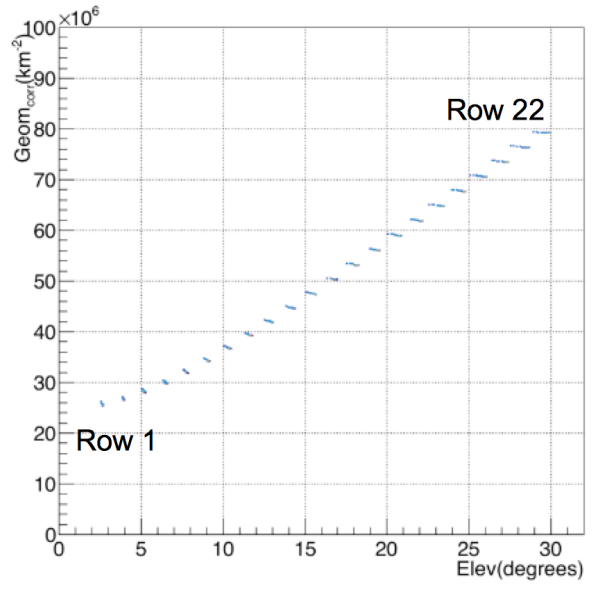

Figure 5. Geometric corrections.

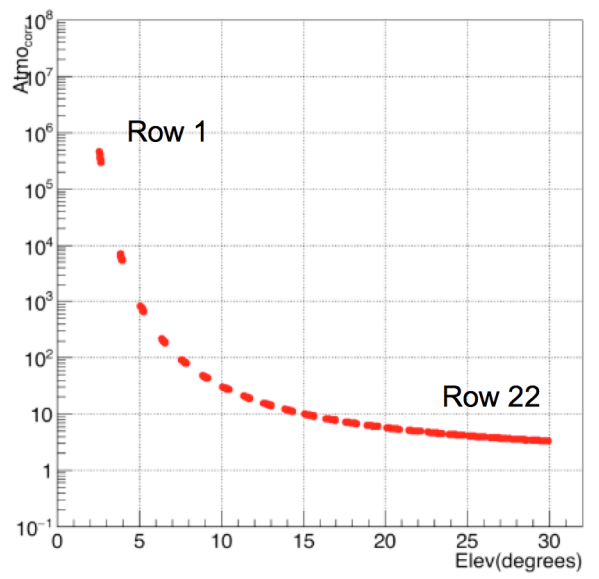

Figure 6. Atmospheric corrections.

\section{Classification of Multiple ELVES}

When more than ten pixels show more than one pulse in the traces, the elve is labeled as multiple and a different type of analysis is performed. The first step consists in plotting the time difference between the two pulses, as shown in Fig.7, that shows one event for each of the four categories of events.

The first two categories are characterized by a constant $\Delta T$ : when it is very short $(<50 \mu \mathrm{s})$ it may be explained as with an interference pattern produced by an EMP emitted by an intracloud lightning, bouncing on the ground. When the gap is larger, the events are either related to the initial breakdown stages of the lightning or to very close return strokes. The third category, events with a $\Delta T$ linearly decreasing with pulse time, appear as single ELVES overlapped to another class of light transient, with a much longer risetime. The fourth group, showing abruptly changing $\Delta T$, identifies ELVES with more than two peaks. This pattern has been proposed as a possible signature of production of terrestrial gamma ray flashes [8]. 


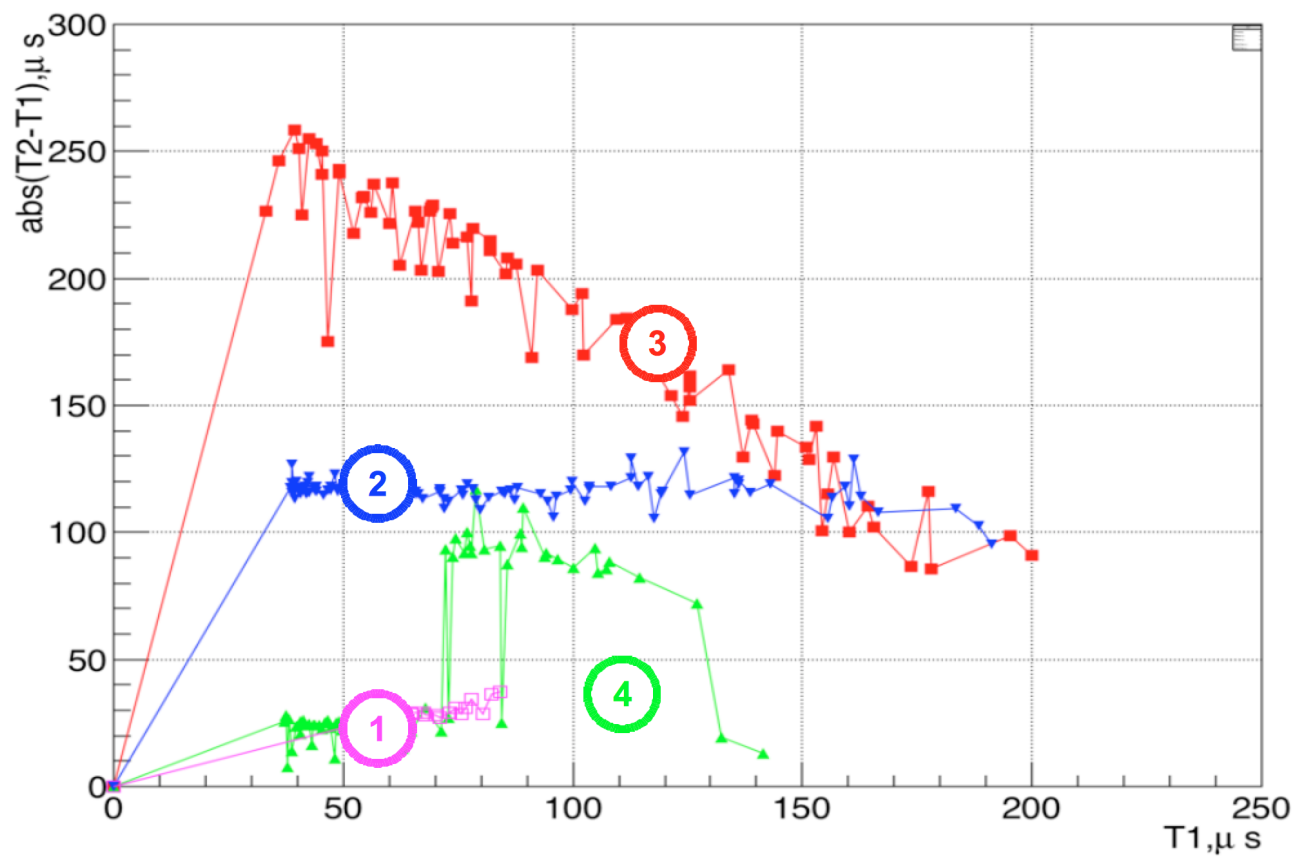

Figure 7. Time difference between two pulses vs time of the first pulse in multiple ELVES.

\section{Conclusions and future prospects}

Since 2013, the Auger Observatory has been taking data with a special trigger devoted to the study of ELVES. The time resolution of our FD gives us unique opportunities for the study of these phenomena, but required to change the length of the trace readout from $10^{3}$ to $9 \times 10^{3}$ bins, in two steps: (a) since 2014, we read $300 \mu$ s in order to see the light emission from the vertical of the causative lightning; (b) since 2017, we can read up to $900 \mu$ s, to study the angular distribution of the entire region of maximum emission around the lightning.

In most cases, we have learned that too simple models are not able to describe the totality of the ELVES events, and larger statistics are required to fully understand the variety of features shown by such interesting phenomena.

In the future months, we are envisaging further upgrades of the TLT selection criteria, to increase its efficiency. We are also planning to enhance our lightning de- tection network, to gain more insights on the mechanisms leading to the production of multiple elves.

\section{References}

[1] U. S. Inan, T. F. Bell, J. V. Rodriguez, Geophys. Res. Lett. 18, (1991) 705

[2] W. L. Boeck et al., Geophys. Res. Lett. 19, (1992) 99

[3] J. Abraham et al. [Pierre Auger Collaboration], Nucl. Instrum. Meth. A 620 (2010) 227

[4] R. Mussa et al. [Pierre Auger Collaboration], Eur. Phys. J. Plus 127 (2012) 94.

[5] A. Aab et al. [Pierre Auger Collaboration], arXiv:1307.5059 [astro-ph.HE].

[6] P. R. Blaes et al., Geophys. Res. Lett.41 (2014) 9182.

[7] F. Kasten and A. T. Young, Applied Optics 28 (1989) 4735.

[8] N. Liu et al., J. Geophys. Res. 122 (2017) 10563. 